\title{
Acute laparoscopic and open sigmoidectomy for perforated diverticulitis: a propensity score-matched cohort
}

\author{
Sandra Vennix ${ }^{1,2} \cdot$ Daniel J. Lips $^{3}$ - Salomone Di Saverio ${ }^{4} \cdot$ Bart A. van Wagensveld $^{5}$. \\ Walter J. Brokelman ${ }^{3}$. Michael F. Gerhards ${ }^{6}$. Anna A. van Geloven ${ }^{7}$. \\ Susan van Dieren ${ }^{8} \cdot$ Johan F. Lange $^{2} \cdot$ Willem A. Bemelman ${ }^{1}$
}

Received: 16 July 2015/Accepted: 17 November 2015/Published online: 17 December 2015

(C) The Author(s) 2015. This article is published with open access at Springerlink.com

\begin{abstract}
Background Hartmann's procedure for perforated diverticulitis can be characterised by high morbidity and mortality rates. While the scientific community focuses on laparoscopic lavage as an alternative for laparotomy, the option of laparoscopic sigmoidectomy seems overlooked. We compared morbidity and hospital stay following acute laparoscopic sigmoidectomy (LS) and open sigmoidectomy (OS) for perforated diverticulitis.

Methods This retrospective cohort parallel to the Ladies trial included patients from 28 Dutch academic or teaching
\end{abstract}

On behalf of the Ladies trial collaborators.

Electronic supplementary material The online version of this article (doi:10.1007/s00464-015-4694-8) contains supplementary material, which is available to authorized users.

Sandra Vennix

s.vennix@amc.nl

1 Department of Surgery, Academic Medical Center, Postbox 22660, 1100 DD Amsterdam, The Netherlands

2 Department of Surgery, Erasmus MC University Medical Center, Rotterdam, The Netherlands

3 Department of Surgery, Jeroen Bosch Hospital, 's-Hertogenbosch, The Netherlands

4 Department of Surgery, Hospital Maggiore, Bologna, Italy

5 Department of Surgery, Sint Lucas Andreas Hospital, Amsterdam, The Netherlands

6 Department of Surgery, OLVG Hospital, Amsterdam, The Netherlands

7 Department of Surgery, Tergooi Hospital, Hilversum, The Netherlands

8 Clinical Research Unit, Academic Medical Center, Amsterdam, The Netherlands hospitals between July 2010 and July 2014. Patients with LS were matched 1:2 to OS using the propensity score for age, gender, previous laparotomy, CRP level, gastrointestinal surgeon, and Hinchey classification.

Results The propensity-matched cohort consisted of 39 patients with LS and 78 patients with OS, selected from a sample of 307 consecutive patients with purulent or faecal perforated diverticulitis. In both groups, $66 \%$ of the patients had Hartmann's procedure and $34 \%$ had primary anastomosis. The hospital stay was shorter following LS (LS 7 vs OS 9 days; $P=0.016$ ), and the postoperative morbidity rate was lower following LS (LS $44 \%$ vs OS $66 \% ; P=0.016$ ). Mortality was low in both groups (LS $3 \%$ vs OS $4 \% ; P=0.685)$. The stoma reversal rate after Hartmann's procedure was higher following laparoscopy, with a probability of being stoma-free at 12 months of 88 and $62 \%$ in the laparoscopic and open groups, respectively $(P=0.019)$. After primary anastomosis, the probability of reversal was $100 \%$ in both groups.

Conclusions In this propensity score-matched cohort, laparoscopic sigmoidectomy is superior to open sigmoidectomy for perforated diverticulitis with regard to postoperative morbidity and hospital stay.

Keywords Diverticulitis - Laparoscopy - Sigmoid resection - Perforated diverticulitis - Propensity score

The classic Hartmann's procedure for perforated diverticulitis can be characterised by high morbidity and mortality rates $[1,2]$. Nowadays, the treatment of has shifted towards less invasive procedures such as laparoscopic lavage, percutaneous drainage, or even conservative management for the milder cases with perforated diverticulitis [3-5]. As laparoscopic lavage has shown to be effective in $75 \%$ of 
the patients with purulent perforated diverticulitis, superior to sigmoidectomy (e.g. Hartmann's procedure or with primary anastomosis) with regard to morbidity and mortality in a randomised controlled trial, it is important to explore other less invasive treatment options besides the classic open Hartmann's procedure [6].

The proven benefit of the laparoscopic approach in the elective setting might even be more pronounced in emergency sigmoidectomy than in the elective setting avoiding in particular abdominal wall complications, e.g. abdominal wound dehiscence, incisional hernia, and wound infection [7-9]. In a systematic review of 5 studies including 104 patients, acute laparoscopic sigmoidectomy for perforated diverticulitis has been shown to be feasible, but comparative studies are lacking [10]. Previously, laparoscopic surgery for acute peritonitis has been under debate due to theoretical concerns of increased bacteraemia and hypercapnia due to the pneumoperitoneum [11, 12]. However, more recent studies suggest even a protective role of the $\mathrm{CO}_{2}$ pneumoperitoneum with a reduced systemic inflammatory response, but similar bacterial translocation [13, 14].

In this propensity-matched cohort, we aim to show a reduction in morbidity and hospital stay following acute laparoscopic sigmoidectomy (LS) compared to open sigmoidectomy (OS) for perforated diverticulitis with generalised peritonitis.

\section{Methods}

\section{Patients}

This retrospective observational cohort consists of consecutive patients with perforated diverticulitis that were not included in the randomised Ladies trial in 28 Dutch teaching hospitals during a 3-year period [6]. Despite the low accrual rate, these patients did not differ in baseline from those randomised within the Ladies trial [6]. The patients were retrospectively identified using the hospital administration code for diverticulitis and acute abdomen, combined with a surgical intervention code to determine the inclusion rate of the Ladies trial. Only the patients requiring acute sigmoidectomy for perforated diverticulitis with purulent or faecal peritonitis have been included. Those with peritoneal lavage or enterostomy without resection have been excluded from analysis, as were those with disease located in other sections than the left colon or sigmoid and those with Hinchey I-II disease or coincidence of fistula. Within the cohort, patients with laparoscopic sigmoidectomy have been identified and were matched 1:2 with patients with open sigmoidectomy based on propensity scores. The term sigmoidectomy is used for both Hartmann's procedure and resection with primary anastomosis; if only one of these two is addressed, the terms Hartmann and primary anastomosis are used. As only anonymous patient data were collected, no ethical approval was required under Dutch law.

\section{Outcomes}

Data have been collected regarding age, gender, BMI (body mass index), American Society of Anesthesiologists (ASA) classification, prescription medication, history of diverticulitis, previous laparotomy, CT diagnosis, preoperative C-reactive protein (CRP) and white blood cell (WBC) count, acute physiology and chronic health evaluation-II (APACHE-II [15]) score, P-POSSUM (Portsmouth Physiology and Operative Severity Score for the enumeration of Mortality and Morbidity [16]) score, and interval from presentation at the emergency department to surgery. Perioperative data have been collected on the Mannheim peritonitis index (MPI) [17], Hinchey classification [18], diagnostic laparoscopy, conversion, intraoperative complications, duration of surgery, and the presence of a gastrointestinal surgeon (defined as a consultant-level surgeon specialised in colorectal or gastrointestinal surgery). Postoperative outcomes assessed were morbidity, scored as Clavien-Dindo $\geq$ I or $\geq$ IIIB [19], mortality, length of hospital stay, ICU admission, and surgical or percutaneous reinterventions. Long-term data were collected on last follow-up, mortality, stoma reversal, and incisional hernia.

\section{Cost analysis}

An economical evaluation was performed to evaluate the costs of laparoscopic versus open sigmoidectomy up to 30 days postoperative or until discharge in the matched cohort. Direct medical costs were estimated using primary data on resource utilisation and included all surgical procedures, including reinterventions and radiological reinterventions, hospital ward stay, and ICU stay. Costs per patient were calculated by multiplying volumes of resources with unit costs. These costs were determined according to the Dutch guidelines of pharmacoeconomic research or based on the tariff of the Academic Medical Center, Amsterdam. Costs were expressed in Euros and inflated when necessary to 2012 .

\section{Statistical analysis}

Before matching, continuous variables were presented as mean with standard deviation (SD) or median with interquartile range (IQR) when appropriate. Discrete variables were presented as numbers of events with percentages. Univariate testing was performed using a $t$ test or 
Mann-Whitney $U$ test when the data were not normally distributed. Pearson's Chi-squared or Fisher's exact test was used for categorical and dichotomous data.

To control for potential confounders, a propensity score was generated for each patient from a multivariable logistic regression model based on baseline variables as independent variables with OS and LS treatment as a binary dependent variable. Those baseline variables in the univariate analysis with a $P$ value $<0.1$ or otherwise of specific clinical interest were added to the model. Sufficient predictive value of the propensity score was defined as an area under the curve (AUC) of $\geq 0.7$ and a Hosmer and Lemeshow (HL) test $P \geq 0.05$.

Each laparoscopic case was matched with replacement to two open cases in a $2: 1$ ratio, with a caliper width of 0.20 standard deviation of the logit of the propensity score using $\mathrm{R}$ statistical software (version 2.13.1). The quality of the match was assessed by comparing the patient characteristics before and after matching as shown in eTable 1 and Table 1.

Data in the matched data set were analysed using a generalised random block design for continuous variables and conditional logistic regression for categorical variables. A two-sided $P$ value of $<0.05$ was considered statistically significant for all tests. Analyses were performed using IBM SPSS version 20.0 software and $\mathrm{R}$ version 2.13.1.

\section{Statistical power}

According to the published literature on elective sigmoidectomy, a $15 \%$ reduction in postoperative major morbidity (Clavien-Dindo grade IIIB or higher) is expected for laparoscopic sigmoidectomy (10\%) compared to open sigmoidectomy (25\%). A power of 0.478 is to be expected, using $\alpha=0.05$ and groups of 39 and 78 patients. Double the sample size would be required to gain a power (1- $\beta$ ) of 0.8 (78 and 156 patients) with a $15 \%$ difference.

\section{Results}

\section{Patient characteristics}

A total of 474 patients treated for perforated diverticulitis could be identified between July 2010 and July 2014
Table 1 Patient demographics in the propensity-matched cohort

\begin{tabular}{lccc}
\hline & Laparoscopic sigmoidectomy & Open sigmoidectomy & $P$ value \\
& $N=39$ & $N=78$ & \\
\hline Age, years & $56.2(14.2)$ & $56.4(13.3)$ & 0.930 \\
Gender, male & $14(35.9)$ & $24(30.8)$ & 0.593 \\
BMI, kg/m ${ }^{2}$ & $25.3(3.5)$ & $27.1(5.8)$ & 0.883 \\
ASA I & $7(22.6)$ & $13(23.6)$ & 0.441 \\
ASA II & $12(38.7)$ & $29(52.7)$ & \\
ASA III & $11(35.5)$ & $11(20.0)$ & \\
ASA IV & $1(3.2)$ & $2(3.6)$ & 0.578 \\
Prescription medication & $19(48.7)$ & $32(43.2)$ & 1.000 \\
History of diverticulitis & $7(17.9)$ & $14(17.9)$ & 1.000 \\
Previous laparotomy & $1(2.6)$ & $65(85.5)$ & 1.000 \\
CT diagnosis & $35(89.7)$ & $166(119)$ & 0.450 \\
CRP level & $158(118)$ & $13.7(6.2)$ & 0.232 \\
WBC count & $15.4(8.9)$ & $6.6(4.2)$ & 0.163 \\
APACHE-II score & $7.4(5.0)$ & $10.5(13.4)$ & 0.935 \\
P-POSSUM predicted mortality $(\%)$ & $9.3(11.7)$ & $67.5(17.5)$ & 0.575 \\
POSSUM predicted morbidity $(\%)$ & $67.7(17.1)$ & $11(6-25)$ & 0.095 \\
Interval to surgery, hours & $11(6-48)$ & $76(97.4)$ & 1.000 \\
Gastrointestinal surgeon present & $38(97.4)$ & $13(16.7)$ & 0.236 \\
MPI score & $19.2(5.3)$ & & 0.608 \\
Hinchey IV & $8(20.5)$ & &
\end{tabular}

Data are mean $(\mathrm{SD})$, number $(\%)$, or median (interquartile range)

$B M I$ body mass index, ASA American Society of Anesthesiologists, CT computed tomography, CRP C-reactive protein, $W B C$ white blood cell, APACHE-II acute physiology and chronic health evaluation-II, POSSUM PS POSSUM-physiology score, POSSUM OS POSSUM-operative score, MPI Mannheim peritonitis index 
Fig. 1 Patient flow chart

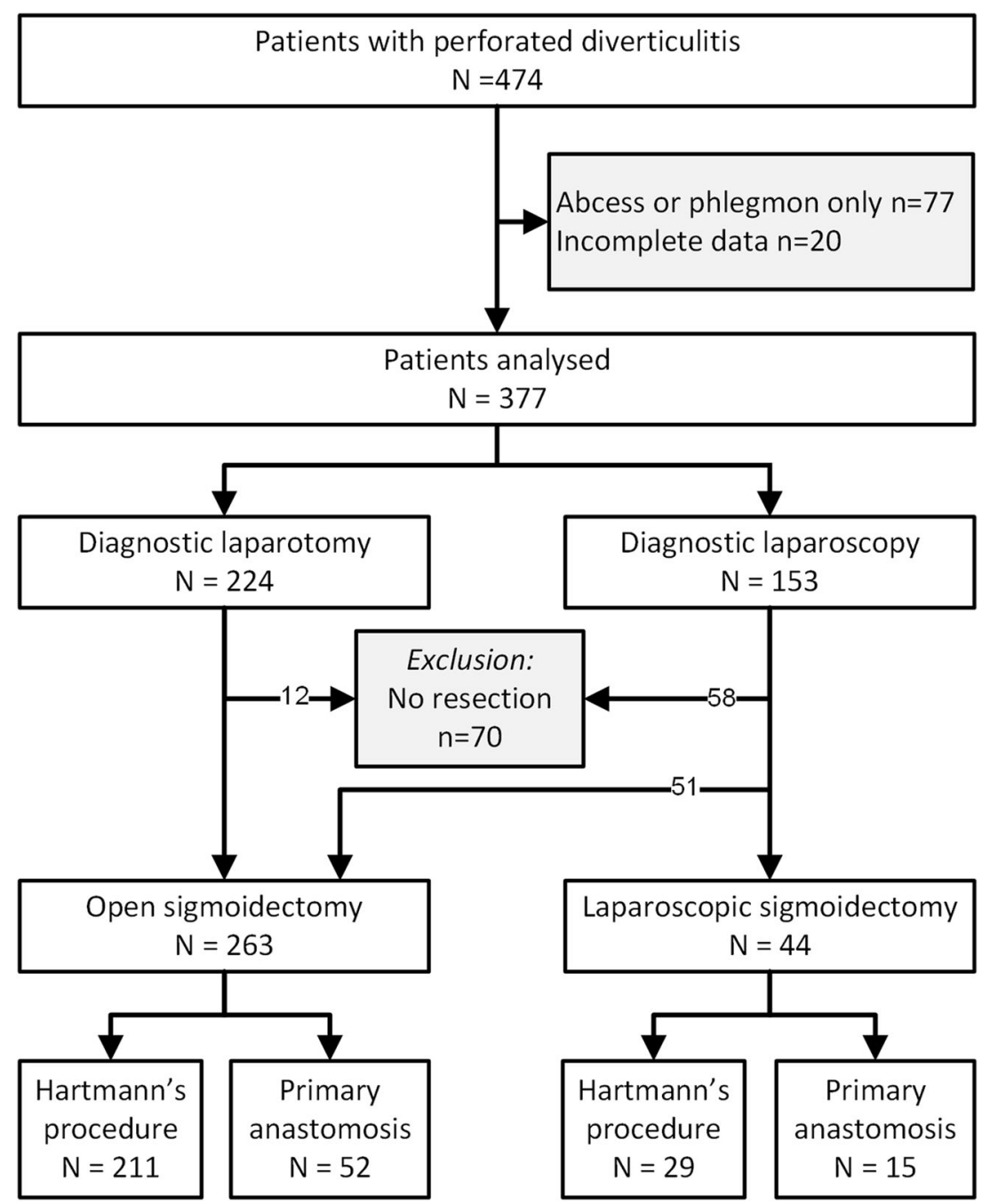

(Fig. 1). Of these, data were available for 377 patients with purulent or faecal peritonitis. A diagnostic laparoscopy (DLS) was performed in $153(41 \%)$ patients, of whom 58 did not undergo resection, 51 were converted to laparotomy, and 44 had laparoscopic sigmoidectomy. From the 224 patients with DL, another 12 were excluded because no resection was performed. In two of the $44(4.5 \%)$ patients in the LS group, laparoscopic resection was attempted, but converted due to adhesions and distended small bowel, and therefore insufficient exposure of the sigmoid perforation.

Patients with LS were identified in 19 out of the 28 hospitals, ranging between 1 and 9 patients each.

In univariate analysis (eTable 1), we found both patients' age and CRP level to be predictive for the choice between LS and OS. Patients with faecal peritonitis and with a higher MPI score were more likely to undergo OS. Specialised gastrointestinal surgeons were more likely to perform LS.

In multivariate analysis with age, gender, previous laparotomy, preoperative CRP level, gastrointestinal surgeon, MPI, and Hinchey classification, only CRP, GI surgeon, and Hinchey classification were found to be predictors for LS (eTable 2). Using all variables from the multivariate analysis, a propensity score for LS or OS was calculated. Only MPI was not included in the propensity score as MPI calculation includes both age and Hinchey classification. The calculated propensity score had a sufficient predictive value with an area under the curve (AUC) of 0.772 (95\% CI 0.704-0.841; HL test, $P=0.309$ ).

\section{Propensity-matched cohort}

The baseline characteristics of the propensity-matched cohort are shown in Table 1. In a 1:2 matching strategy, 39 patients with laparoscopic sigmoidectomy were matched to 78 patients with open sigmoidectomy. Due to the replacements during the automatic matching process, the control group consisted of 59 unique patients with open sigmoidectomy and 19 duplicates. Following matching, no differences persisted in any of the matched variables as 
expected. In addition, no differences were found between the groups with regard to all other variables such as the ASA classification and preoperative POSSUM and APACHE scores. Sigmoidectomy was performed as Hartmann's procedure in $66 \%$ of patients and primary anastomosis in $34 \%$. Of these patients with primary anastomosis, $8 / 13$ had a deviating ileostomy following LS compared to $12 / 27$ in the OS group. The surgical duration for LS was longer with 127 min compared to $97 \mathrm{~min}$ for OS $(P=0.003)$ (Table 2).

Intraoperative complications occurred in 1 patient during LS and two patients during OS. Two patients had an intraoperative bleeding, and in one patient, the stapler donuts of the attempted primary anastomosis were incomplete and an end colostomy was created instead. Intraoperative blood loss was reported as $<100 \mathrm{ml}$ in 14 (74\%) patients in LS and $15(42 \%)$ in the OS $(P=0.117)$. Following acute surgery, $11(37 \%)$ LS patients and $28(50 \%)$ OS patients were admitted to the ICU.

\section{Postoperative outcomes}

Laparoscopic sigmoidectomy resulted in a shorter hospital stay compared to OS ( 7 versus 9 days, $P=0.016$ ). The inhospital overall morbidity rate was lower following LS $(P=0.016)$. No significant difference was found with regard to in-hospital mortality and reinterventions as shown in Table 2. In-hospital mortality was reported in 1 (3\%) patient following LS and in 3 following OS (4\%). Overall morbidity was lower in the laparoscopic group. No difference was seen for surgical reinterventions (LS $5 \%$ vs OS $9 \% ; P=0.485$ ) or severe morbidity (Clavien-Dindo $\geq \mathrm{IIIB}$, LS $13 \%$ vs OS $20 \%$; $P=0.253$ ). A significant reduction in wound infections was found (LS $3 \%$ vs OS $29 \% ; P=0.009$, eTable 3). Anastomotic leakage occurred in one patient following LS and was treated by relaparotomy and loop ileostomy.

In a subgroup analysis, laparoscopic and open Hartmann's procedure were compared, showing $8 \%$ (OS) versus $4 \%$ (LS) mortality $(P=0.476)$, and severe morbidity occurred in $39 \%$ (OS) versus $15 \%$ (LS) $(P=0.037)$. Postoperative hospital stay was $12(8-21)$ days versus $8(5-15)$ days for open and laparoscopic Hartmann's, respectively $(P=0.006)$. In the primary anastomosis group, the mortality and severe morbidity rate were $0 \%$ in both the laparoscopic and open group. Postoperative hospital stay was 8 (7-9) days versus 7 (6-10) days for open and laparoscopic primary anastomosis, respectively $(P=0.391)$.

The calculated costs were lower for laparoscopic sigmoidectomy (mean difference $€-8$ 336, $95 \% \mathrm{CI} €-16$ 113 to $€ 588 ; P=0.031$ ); these costs included only the direct costs for the primary hospital admission. For subgroups of costs, particularly the costs for ICU stay were five times higher for open sigmoidectomy $(P=0.022)$ (Table 3).

\section{Long-term outcomes}

The median length of follow-up was shorter in the LS group with 8 (IQR 5-12) months compared to 16 (IQR $7-28)$ months in the OS group $(P<0.001)$ as more patients had LS later in the study period (eTable 4). Stoma reversal was associated with Hartmann's procedure or primary anastomosis and not with laparoscopic or open surgery in multivariable regression analysis (data not shown). Therefore, Kaplan-Meier analysis was performed
Table 2 Surgical and shortterm postoperative outcomes in the propensity-matched cohort

\begin{tabular}{lccc}
\hline & Laparoscopic sigmoidectomy & Open sigmoidectomy & $P$ value \\
& $N=39$ & 96 & \\
\hline Duration of surgery, minutes & $127(105-159)$ & $96.5(87-120)$ & 0.003 \\
Hartmann's procedure & $26(66.7)$ & $51(65.4)$ & 0.890 \\
Primary anastomosis & $13(33.3)$ & $27(34.6)$ & \\
Ileostomy rate & $8 / 13(61.5)$ & $12 / 27(44.4)$ & 0.597 \\
Postoperative ICU admission & $11(36.7)$ & $28(50.0)$ & 0.305 \\
In-hospital mortality & $1(2.6)$ & $3(3.9)$ & 0.685 \\
In-hospital overall morbidity & $17(43.6)$ & $51(66.2)$ & 0.016 \\
In-hospital severe morbidity $(>$ IIIB) & $5(12.8)$ & $15(19.5)$ & 0.253 \\
Reinterventions & $5(12.8)$ & $7(19.5)$ & 0.739 \\
$\quad$ Surgical reinterventions & $2(5.1)$ & $10(13.0)$ & 0.485 \\
Percutaneous reinterventions & $3(7.7)$ & $9(7-14)$ & 0.419 \\
Postoperative hospital stay, days & $7(5-13)$ & 0.016 \\
\hline
\end{tabular}

Data are mean (SD), number (\%), or median (interquartile range). ICU intensive care unit. Severe morbidity defined as Clavien-Dindo $\geq$ IIIB 
Table 3 Costs calculation based on short-term data only (Euro)

\begin{tabular}{lllllll}
\hline & \multicolumn{2}{l}{ Lap sigmoidectomy } & & \multicolumn{2}{l}{ Open sigmoidectomy } & \multirow{2}{*}{$P$ value } \\
\cline { 2 - 3 } & Units & Total costs & & Units & Total costs & \\
\hline Primary surgery & 39 & 153,426 & & 78 & 241,878 & - \\
Days at hospital ward & 355 & 172,328 & & 846 & 410,674 & 0.108 \\
Days at ICU & 38 & 88,114 & & 350 & 806,942 & 0.022 \\
Percutaneous drainage & 3 & 486 & & 10 & 1619 & 0.418 \\
Surgical reinterventions & 3 & 9589 & & 8 & 25,571 & 0.698 \\
Total & & 423,943 & & & $1,486,684$ & \\
Total per patient, Euro & $10,870(4710-17,031)$ & & $19,209(14,850-23,563)$ & 0.031 \\
\hline
\end{tabular}

Total per patient as mean (95\% confidence interval). Three surgical reinterventions in two patients, and eight reinterventions in seven patients

$I C U$ intensive care unit for four groups: open Hartmann's, open primary anastomosis, laparoscopic Hartmann's, and laparoscopic primary anastomosis with probabilities of being stoma-free at 12 months of $0.64,1.00,0.88$, and 1.00 , respectively $(P<0.001$, log rank test; Fig. 2 and eFigure 1). Colostomies in the laparoscopic group were reversed more often using the laparoscopic technique (12/13 LS, 4/28 OS; $P<0.001)$.

\section{Discussion}

This first comparative study between open and laparoscopic sigmoidectomy shows laparoscopic sigmoidectomy for perforated diverticulitis to be superior to open sigmoidectomy with regard to morbidity and hospital stay. Laparoscopic sigmoidectomy is safe and feasible as shown by the low conversion rate and postoperative mortality that

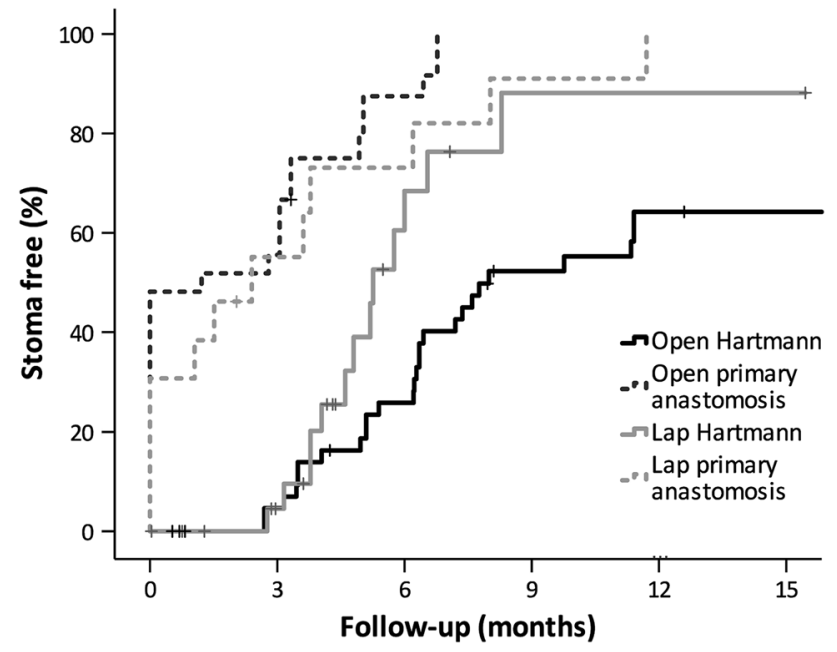

Fig. 2 Probability of being stoma-free. Generated by Kaplan-Meier method. Log rank for all groups $P<0.001$. Log rank for Hartmann's lap versus open $P=0.019$. Log rank for primary anastomosis lap versus open $P=0.272$ did not differ significantly compared to OS. The lower morbidity and hospital stay resulted in reduced costs per patient in the laparoscopic group. Stoma closure after Hartmann's procedure occurred more often after a laparoscopic approach.

Up to now, only a few small non-comparative series have been described regarding laparoscopic sigmoidectomy for perforated diverticulitis [10]. Favourable results were described in these selected patients, especially in comparison with the older open sigmoidectomy series. Another series by Turley et al. [20] compared two groups of 67 patients in a propensity-matched cohort out of 1186 patients with emergency Hartmann's procedure for diverticulitis. No statistically significant differences in postoperative morbidity (30 vs $25 \%$ ), mortality (4.5 and $3.0 \%$ ), and hospital stay ( 8 vs 6 days) were shown between open and laparoscopic surgery. A limitation of that study is the unclear indication for surgery in the included patients, which was not limited to Hinchey III and IV perforated diverticulitis.

In the EAES guidelines for emergency abdominal surgery, laparoscopic sigmoidectomy is described as a feasible option in experienced hands [21]. However, the cited paper by Zdichavsky et al. [22] describes a semi-acute series after failed medical management of low grades of perforated diverticulitis. Although a slightly different population, these series do support the feasibility of laparoscopic sigmoidectomy in a contaminated abdomen.

Although overall postoperative morbidity was higher following open sigmoidectomy, only the difference in wound infection rate was statistically significant and made up for the complete $20 \%$ difference. However, even without the wound infections, the total number of complications was higher following open sigmoidectomy (16 vs 73 surgical events and 23 vs 96 total events in 39 and 78 patients).

Stoma reversal did not differ between LS and OS after primary anastomosis; the probability of reversal was $100 \%$ in both groups, while $38 \%$ of the patients after LS and 
$56 \%$ after OS never had an ileostomy. This finding is not surprising, because closure of the defunctioning ileostomy can be done without laparotomy. The initial approach, either laparoscopic or open, does not affect the reversal rate for this reason [23, 24]. Following Hartmann's procedure, the probability of reversal for LS was $87 \%$ compared to $63 \%$ for OS. These data are reflected in previous study on acute Hartmann's procedure, with a reversal rate of $72 \%$ after LS and $57 \%$ after OS in less selected patient populations $[23,25]$.

The main concern regarding laparoscopic surgery in general peritonitis is the risk of damage to the distended and vulnerable small bowel. A recent systematic review reported $64 \%$ success of laparoscopic treatment in 2005 patients with small bowel obstruction. About $10 \%$ of the conversions were due to iatrogenic injury and $7.6 \%$ due to inadequate exposure [26]. Even a small bowel diameter $>4 \mathrm{~cm}$ was not considered to be a contraindication for laparoscopy [27].

This study used a propensity-matched design to evaluate differences between both groups. This design allows for a correction for bias introduced by selection of patients, improving the reliability of the presented outcomes. This design has been shown to provide similar treatment effects compared to randomised studies and therefore can be relied upon when randomised trials are not feasible [28].

The present study has some limitations. First, its retrospective and non-randomised design might have introduced selection and reporting bias. Although all patient records were fully searched for outcomes, the registration might be incomplete compared to proper prospective registration. Second, the small proportion of patients with laparoscopic sigmoidectomy is likely to be selected based on favourable patient or disease characteristics, in combination with surgeon's preferences. The patients selected for laparoscopy had a lower preoperative CRP level and a non-significant difference in age and ASA classification compared to open sigmoidectomy. Although only patients with Hinchey III and IV disease and no Hinchey II disease have been included, these results apply especially to patients with similar characteristics to those in this matched cohort.

After diagnostic laparoscopy, in 44 patients the procedure was continued by laparoscopy, while 51 patients were converted to laparotomy. Due to the retrospective nature of this study, the reason and moment of conversion remain unclear due to lack of standardised reporting. Some were converted before the diagnosis was clear and others upon diagnosing perforated diverticulitis. In two patients, conversion was described after attempting laparoscopic resection, and therefore recorded as conversion within the laparoscopic group, and analysed according to intention to treat.

\section{Conclusions}

In this propensity score-matched cohort, laparoscopic sigmoidectomy is superior to open sigmoidectomy for perforated diverticulitis with regard to postoperative morbidity and hospital stay. Although the groups are matched, the results should be interpreted with caution as the cohort consists of selected patients with more favourable baseline characteristics compared to the complete group and surgery was performed by experienced gastrointestinal surgeons.

Acknowledgments The investigators and the participating centres in this study are listed below in alphabetical order of their location. Hospitals are located in the Netherlands unless stated otherwise. All these clinical investigators have been involved in the data collection for this study.

Funding This study was performed within the Ladies trial which was funded by a ZonMW Dutch governmental Grant (171002213).

Ladies trial collaborators ECJ Consten (Department of Surgery, Meander Medical Centre, Amersfoort); WA Bemelman (Department of Surgery, Academic Medical Center, Amsterdam); MF Gerhards (Department of Surgery, Onze Lieve Vrouwe Hospital, Amsterdam); BA van Wagensveld (Department of Surgery, St Lucas Andreas Hospital, Amsterdam); HA Cense (Department of Surgery, Rode Kruis Hospital, Beverwijk); RMPH Crolla (Department of Surgery, Amphia Hospital, Breda); EJR de Graaf (Department of Surgery, IJsselland Hospital, Capelle a/d IJssel); TM Karsten (Department of Surgery, Reinier de Graaf Hospital, Delft); HA Prins (Department of Surgery, Jeroen Bosch Hospital, Den Bosch); JAB van der Hoeven (Department of Surgery, Albert Schweitzer Hospital, Dordrecht); PM Kruyt (Department of Surgery, Gelderse Vallei Hospital, Ede); SW Nienhuijs (Department of Surgery, Catharina Hospital, Eindhoven); EB van Duyn (Department of Surgery, Medical Spectrum Twente, Enschede); DJ Swank (Department of Surgery, Groene Hart Hospital, Gouda); HBAC Stockmann (Department of Surgery, Kennemer Hospital, Haarlem); HJ Belgers (Department of Surgery, Atrium Medical Centre, Heerlen); AAW van Geloven (Department of Surgery, Tergooi Hospital, Hilversum); QAJ Eijsbouts (Department of Surgery, Spaarne Hospital, Hoofddorp); M.J.P.M. Govaert (Department of Surgery, Westfries Hospital, Hoorn); MJ Wiezer (Department of Surgery, St Antonius Hospital, Nieuwegein); JF Lange (Department of Surgery, Erasmus Medical Centre University Hospital, Rotterdam); GHH Mannaerts (Department of Surgery, St Franciscus Hospital, Rotterdam); AGM Hoofwijk (Department of Surgery, Orbis Medical Centre, Sittard); WH Steup (Department of Surgery, Haga Hospital, The Hague); DDE Zimmerman (Department of Surgery, Twee Steden Hospital, Tilburg); WMU van Grevenstein (Department of Surgery, University Medical Centre, Utrecht); JLM Konsten (Department of Surgery, VieCuri Hospital, Venlo); EGJM Pierik (Department of Surgery, Isala Hospital, Zwolle); AJL D'Hoore (Department of Surgery, University Hospital, Leuven, Belgium); S Di Saverio (Department of Surgery, Hospital Maggiore, Bologna, Italy).

\section{Compliance with ethical standards}

Disclosures SV, DJL, SDS, BAW, WJB, MFG, AAvG, SvD, JFL, and $\mathrm{WAB}$ have no conflicts of interest or financial ties to disclose.

Open Access This article is distributed under the terms of the Creative Commons Attribution 4.0 International License (http://crea tivecommons.org/licenses/by/4.0/), which permits unrestricted use, 
distribution, and reproduction in any medium, provided you give appropriate credit to the original author(s) and the source, provide a link to the Creative Commons license, and indicate if changes were made.

\section{References}

1. Vermeulen J, Akkersdijk GP, Gosselink MP, Hop WC, Mannaerts GH, van der Harst E, Coene PP, Weidema WF, Lange JF (2007) Outcome after emergency surgery for acute perforated diverticulitis in 200 cases. Dig Surg 24:361-366

2. Morris CR, Harvey IM, Stebbings WS, Hart AR (2008) Incidence of perforated diverticulitis and risk factors for death in a UK population. Br J Surg 95:876-881

3. McDermott FD, Collins D, Heeney A, Winter DC (2014) Minimally invasive and surgical management strategies tailored to the severity of acute diverticulitis. Br J Surg 101:e90-e99

4. Sallinen VJ, Mentula PJ, Leppaniemi AK (2014) Nonoperative management of perforated diverticulitis with extraluminal air is safe and effective in selected patients. Dis Colon Rectum 57:875-881

5. Costi R, Cauchy F, Le Bian A, Honart JF, Creuze N, Smadja C (2012) Challenging a classic myth: pneumoperitoneum associated with acute diverticulitis is not an indication for open or laparoscopic emergency surgery in hemodynamically stable patients. A 10-year experience with a nonoperative treatment. Surg Endosc 26:2061-2071

6. Vennix S, Musters GD, Mulder IM, Swank HA, Consten EC, Belgers EH, van Geloven AA, Gerhards MF, Govaert MJ, van Grevenstein WM, Hoofwijk AG, Kruyt PM, Nienhuijs SW, Boermeester MA, Vermeulen J, van Dieren S, Lange JF, Bemelman WA, Ladies trial c (2015) Laparoscopic peritoneal lavage or sigmoidectomy for perforated diverticulitis with purulent peritonitis: a multicentre, parallel-group, randomised, openlabel trial. Lancet 386:1269-1277

7. Phillips EH, Franklin M, Carroll BJ, Fallas MJ, Ramos R, Rosenthal D (1992) Laparoscopic colectomy. Ann Surg 216:703-707

8. Kuhry E, Schwenk WF, Gaupset R, Romild U, Bonjer HJ (2008) Long-term results of laparoscopic colorectal cancer resection. Cochrane Database Syst Rev 2008:CD003432. doi:10.1002/ 14651858.CD003432.pub2

9. Klarenbeek BR, Veenhof AA, Bergamaschi R, van der Peet DL, van den Broek WT, de Lange ES, Bemelman WA, Heres P, Lacy AM, Engel AF, Cuesta MA (2009) Laparoscopic sigmoid resection for diverticulitis decreases major morbidity rates: a randomized control trial: short-term results of the Sigma Trial. Ann Surg 249:39-44

10. Vennix S, Boersema GS, Buskens CJ, Menon AG, Tanis PJ, Lange JF, Bemelman WA (2015) Emergency laparoscopic sigmoidectomy for perforated diverticulitis with generalised peritonitis: a systematic review. Dig Surg 33:1-7

11. Navez B, Tassetti V, Scohy JJ, Mutter D, Guiot P, Evrard S, Marescaux J (1998) Laparoscopic management of acute peritonitis. Br J Surg 85:32-36

12. Neudecker J, Sauerland S, Neugebauer E, Bergamaschi R, Bonjer HJ, Cuschieri A, Fuchs KH, Jacobi C, Jansen FW, Koivusalo AM, Lacy A, McMahon MJ, Millat B, Schwenk W (2002) The European Association for Endoscopic Surgery clinical practice guideline on the pneumoperitoneum for laparoscopic surgery. Surg Endosc 16:1121-1143

13. Machado MC, Coelho AM, Martins JO, Sampietre SN, Molan NA, Patzina RA, Machado MA, Jancar S (2010) CO2 abdominal insufflation decreases local and systemic inflammatory response in experimental acute pancreatitis. Pancreas 39:175-181
14. Wang G, Wu R, Guo F, Liu W, Chen X, Yu Q (2014) Effects of carbon dioxide pneumoperitoneum on the inflammatory response and bacterial translocation in intraabdominal infection. $\mathrm{J}$ Laparoendosc Adv Surg Tech A 24:199-204

15. Knaus WA, Draper EA, Wagner DP, Zimmerman JE (1985) APACHE II: a severity of disease classification system. Crit Care Med 13:818-829

16. Prytherch DR, Whiteley MS, Higgins B, Weaver PC, Prout WG, Powell SJ (1998) POSSUM and Portsmouth POSSUM for predicting mortality. Physiological and operative severity score for the enUmeration of mortality and morbidity. $\mathrm{Br} \mathrm{J}$ Surg 85:1217-1220

17. Linder MM, Wacha H, Feldmann U, Wesch G, Streifensand RA, Gundlach E (1987) The Mannheim peritonitis index — an Instrument for the intraoperative prognosis of peritonitis. Chirurg 58:84-92

18. Hinchey EJ, Schaal PG, Richards GK (1978) Treatment of perforated diverticular disease of the colon. Adv Surg 12:85-109

19. Dindo D, Demartines N, Clavien PA (2004) Classification of surgical complications: a new proposal with evaluation in a cohort of 6336 patients and results of a survey. Ann Surg 240:205-213

20. Turley RS, Barbas AS, Lidsky ME, Mantyh CR, Migaly J, Scarborough JE (2013) Laparoscopic versus open Hartmann procedure for the emergency treatment of diverticulitis: a propensity-matched analysis. Dis Colon Rectum 56:72-82

21. Agresta F, Ansaloni L, Baiocchi GL, Bergamini C, Campanile FC, Carlucci M, Cocorullo G, Corradi A, Franzato B, Lupo M, Mandala V, Mirabella A, Pernazza G, Piccoli M, Staudacher C, Vettoretto N, Zago M, Lettieri E, Levati A, Pietrini D, Scaglione M, De Masi S, De Placido G, Francucci M, Rasi M, Fingerhut A, Uranus S, Garattini S (2012) Laparoscopic approach to acute abdomen from the Consensus Development Conference of the Societa Italiana di Chirurgia Endoscopica e nuove tecnologie (SICE), Associazione Chirurghi Ospedalieri Italiani (ACOI), Societa Italiana di Chirurgia (SIC), Societa Italiana di Chirurgia d'Urgenza e del Trauma (SICUT), Societa Italiana di Chirurgia nell'Ospedalita Privata (SICOP), and the European Association for Endoscopic Surgery (EAES). Surg Endosc 26:2134-2164

22. Zdichavsky M, Kratt T, Stuker D, Meile T, Feilitzsch MV, Wichmann D, Konigsrainer A (2013) Acute and elective laparoscopic resection for complicated sigmoid diverticulitis: clinical and histological outcome. J Gastrointest Surg 17:1966-1971

23. Oberkofler CE, Rickenbacher A, Raptis DA, Lehmann K, Villiger P, Buchli C, Grieder F, Gelpke H, Decurtins M, Tempia-Caliera AA, Demartines N, Hahnloser D, Clavien PA, Breitenstein S (2012) A multicenter randomized clinical trial of primary anastomosis or Hartmann's procedure for perforated left colonic diverticulitis with purulent or fecal peritonitis. Ann Surg 256:819-826 (discussion 826-817)

24. Kaidar-Person O, Person B, Wexner SD (2005) Complications of construction and closure of temporary loop ileostomy. J Am Coll Surg 201:759-773

25. Liang S, Russek K, Franklin ME Jr (2012) Damage control strategy for the management of perforated diverticulitis with generalized peritonitis: laparoscopic lavage and drainage vs. laparoscopic Hartmann's procedure. Surg Endosc 26:2835-2842

26. O'Connor DB, Winter DC (2012) The role of laparoscopy in the management of acute small-bowel obstruction: a review of over 2000 cases. Surg Endosc Other Interv Tech 26:12-17

27. Pearl JP, Marks JM, Hardacre JM, Ponsky JL, Delaney CP, Rosen MJ (2008) Laparoscopic treatment of complex small bowel obstruction: is it safe? Surg Innov 15:110-113

28. Lonjon G, Boutron I, Trinquart L, Ahmad N, Aim F, Nizard R, Ravaud P (2014) Comparison of treatment effect estimates from prospective nonrandomized studies with propensity score analysis and randomized controlled trials of surgical procedures. Ann Surg 259:18-25 